
\title{
$\begin{array}{ll}\text { Research Square } & \begin{array}{l}\text { Preprints are preliminary reports that have not undergone peer review. } \\ \text { They should not be considered conclusive, used to inform clinical practice, } \\ \text { or referenced by the media as validated information. }\end{array}\end{array}$
}

\section{Impact of Different Ventilation Strategies on Gas Exchanges and Circulation During Prolonged Mechanical Cardio-Pulmonary Resuscitation in a Porcine Model}

\author{
Caroline FRITZ \\ INSERM U1116 \\ Deborah JAEGER \\ INSERM U1116 \\ Yun LUO \\ INSERM U1116 \\ Emilie LARDENOIS \\ Universite de Lorraine Faculte de Medecine \\ Bilal BADAT \\ Air Liquide Healthcare \\ Florian Eric ROQUET \\ APHP: Assistance Publique - Hopitaux de Paris \\ Marceau RIGOLLOT \\ Air Liquide Healthcare \\ Antoine KIMMOUN \\ INSERM U1116 \\ N'Guyen TRAN \\ Universite de Lorraine Faculte de Medecine \\ Jean-Christophe RICHARD \\ $\mathrm{CH}$ Annecy: Centre Hospitalier Annecy Genevois \\ Tahar CHOUIHED \\ INSERM U1116 \\ Bruno LEVY ( $\nabla$ b.levy@chu-nancy.fr) \\ INSERM U 1116 https://orcid.org/0000-0002-7630-2908
}

\section{Research}

Keywords: Ventilation methods, Cardiac arrest, cardio-pulmonary resuscitation

Posted Date: August 5th, 2021

DOI: https://doi.org/10.21203/rs.3.rs-751442/v1

License: @ (i) This work is licensed under a Creative Commons Attribution 4.0 International License. Read Full License

Version of Record: A version of this preprint was published at Shock on October 27th, 2021. See the published version at https://doi.org/10.1097/SHK.0000000000001880. 


\section{Abstract}

Background:

Optimal ventilation during cardio-pulmonary resuscitation (CPR) is still controversial. Ventilation is expected to provide sufficient arterial oxygen content and adequate carbon dioxide removal, while minimizing the risk of circulatory impairment. The objective of the present study was to compare three ventilation strategies in a porcine model during mechanical continuous chest compressions (CCC) according to arterial oxygenation and hemodynamic impact.

\section{Method}

Ventricular fibrillation was induced and followed by five no-flow minutes and thirty low-flow minutes resuscitation with mechanical-CCC without vasopressive drugs administration. Three groups of eight Landras pig were randomized according to the ventilation strategy: i) Standard volume-control mode (SD-group); ii) bi-level pressure mode (CPV-group); iii) continuous insufflation with Boussignac Cardiac-Arrest Device (BC-group). We assessed i) arterial blood gases, ii) macro hemodynamics, iii) tissular cerebral macro and micro-circulation and iiii) airway pressure, minute ventilation at baseline and every five minutes during the protocol.

\section{Results:}

Arterial PaO2 level was higher at each measurement time in SD-group ( $>200 \mathrm{mmHg}$ ) compare to CPV-group and BC-group ( $<<0.01)$. In BC-group, arterial PaCO2 level was significantly higher $(>90 \mathrm{mmHg})$ than in SD and CPV groups $(p<0.01)$. There was no difference between groups concerning hemodynamic parameters, cerebral perfusion and microcirculation.

\section{Conclusion:}

Ventilation modalities in this porcine model of prolonged CPR influence oxygenation and decarboxylation without impairing circulation and cerebral perfusion.

\section{Introduction}

Neurologic prognosis of cardiac arrest is highly dependent of the quality of CPR. The International Liaison Committee on Resuscitation strongly encourages high-quality chest compressions (CC). Nevertheless, ventilation settings during CPR management are still controversial (1-3). Ventilation is expected to provide sufficient arterial oxygenation and adequate carbon dioxide removal, while minimizing the risk of circulatory impairment, with imprecise targets during CRP. The latest ERC guidelines called for $100 \% \mathrm{FiO}_{2}$ use during CPR and a saturation of at least $94 \%$ in post cardiac arrest (4). Ventilation strategies may impact gas exchanges and circulation differently (5), especially when CPR with mechanical chest compression is prolonged (6). Practices in the field differ according to the teams (7) with different underlying concepts.

For advance life support, international guidelines emphasize basic volume-mode ventilation (Tidal volume at $6 \mathrm{~mL}$ of predictable body weight with a respiratory rate of $10 . \mathrm{min}^{-1}$ ) with no positive end-expiratory pressure (PEEP). A bi-level pressure-mode ventilation approach has been developed through CPV mode (CPV Monnal T60, Air Liquide Medical System, Antony, France). Insufflation is synchronized with $\mathrm{CC}$ with an upper pressure set at $20 \mathrm{cmH}_{2} \mathrm{O}$ and a lower pressure set at $5 \mathrm{cmH}_{2} \mathrm{O}$. This pressure mode ventilation is synchronized with $\mathrm{CC}$, limiting hyperventilation or high airway pressure and keeping airways open (8). Finally, continuous oxygen insufflation (COI) is an old concept developed to avoid interruption of CC during CPR (10-12). The Boussignac Cardiac Arrest Device (B-CARD, Vygon, Ecouen, France) (13) actively delivers oxygen at $15 \mathrm{~L}^{\mathrm{m}} \mathrm{min}^{-1}$. Potential benefits of this device on hemodynamics is debated (14). Since the B-CARD does not provide ventilation, we will use it as a model to study the effects of oxygenation without ventilation. Finally the effect on intrathoracic pressure of $\mathrm{COI}$ differs according to experimental studies, either positive or negative $(1,12)$.

Therefore, the objective of this study was to compare three ventilation strategies used on the field (Standard, CPV and B-CARD) based on results on arterial oxygenation and decarboxylation, systemic and cerebral hemodynamics in a porcine model of prolonged CA under mechanical continuous chest compressions (MCCC).

\section{Methods}

\section{Ethics approval}

All experiments were reviewed and approved by the Nancy University Ethics Committee for Animal Experimentation (APAFIS number 101992017061212039981v1). The procedure for the care and sacrifices of study animals was in accordance with the European Community Standards on the Care and Use of Laboratory Animals.

\section{Animal model.}

Animal preparation. Twenty-four domestic male pigs (Landrace) weighing 44 to $65 \mathrm{~kg}$ were acclimated to the animal facilities for four to seven days and fasted overnight prior to experimentation with free access to water. All animals were premedicated with an intramuscular injection of ketamine (15 mg.kg ${ }^{-1}$ Ketalar, Parke-Davis, Courbevoie, France) and midazolam ( $0.1 \mathrm{mg} . \mathrm{kg}^{-1}$, Hypnovel; Produits Roche, Neuilly sur Seine, France). Anaesthesia was induced as previously published (15) via the lateral auricular vein with an intravenous bolus of propofol (1 mg. $\mathrm{kg}^{-1}$, Propofol-lipuro 1\%, B. Braun, Melsungen AG, Germany). Animals were intubated (TeleflexIsis 7.5 I.D. mm, Teleflex Medical, Athlone, Ireland) and mechanically ventilated (Evita 1 Dura, Dräger, Luebeck, 
Germany) in assisted-controlled mode (30\% Oxygen, tidal volume $10 \mathrm{mg} \cdot \mathrm{kg}^{-1}$ and respiratory rate 12). Anaesthesia was maintained with continuous infusion of sufentanil ( $0.2 \mu \mathrm{g} \cdot \mathrm{kg}^{-1} \cdot \mathrm{min}^{-1}$, Sufentanil, Mylan, Canonsburg, Pennsylvania, USA) and propofol $\left(7 \mathrm{mg} \cdot \mathrm{kg}^{-1} \cdot \mathrm{h}^{-1}\right.$, propofol-lipuro $2 \%$, B. Braun Melsungen AG, Germany and cisatracurium (0.9 mg. $\mathrm{kg}^{-1} \cdot \mathrm{h}^{-1}$, Nimbex, GlaxoSmithKline, Brentford, Middlesex, UK). Unfractionated heparin (10 Ul.kg ${ }^{-1}$, Heparine Sodique Choay, Sanofi-Aventis, Paris, France) was administered to avoid catheter clotting. Normovolemia was maintained by administering NaCl $0.9 \%\left(10 \mathrm{ml}^{\mathrm{kg}} \mathrm{kg}^{-1} . \mathrm{h}^{-1}\right.$, Osalia SALF, SpA Laboratiorio Farmacologico, Cenate Sotto, Italie). Body temperature was controlled for a core temperature at $38^{\circ} \mathrm{C}$. All experiment occurred at the same moment during circadian cycle (during morning time).

Specifically, for the procedure, after dissection of neck vessels, a venous catheter was introduced in the right external jugular vein (Swan-Ganz catheter introductor, Edwards Lifesciences, USA). A catheter was introduced in the right femoral artery (Seldicath, Plastimed Prodimed, France) to monitor systemic blood pressure. Venous and arterial catheter were connected to two pressure transducers (Emka usbACQ, usbAMP ; Emka technologies SAS, Paris, France). A transit time flow probe (Transonic Systems Inc, USA) was placed around the left carotid artery.

In the corresponding region of the left hemisphere, an intracranial pressure probe (Intracranial scisense catheter, Transonic scisense Inc, London, Canada) was inserted after trepanation (Codman Disposable perforator $14 \mathrm{~mm}$, Johnson \& Johnson Medical Ltd, Wokingham, UK). NIRS cerebral sensors were fixed on forehead (Masimo SET O3 Sensor, Masimo Corporation CA, USA). NIRS peripheral sensor was fixed on the anterior left leg after shaving (Inspectra StO2 sensor thenar Model 1615, Hutchinson Technology Inc,Hutchinson, MN 55350 USA). The head remained in standard horizontal supine position during the protocol.

Flow and Airway Pressure (Paw) were measured using a Pneumotachograph (Pneumotachometer, Hans Rudolph inc, Shawnee, USA) positioned proximally to the endotracheal tube and connected to two pressure transducers (Emka usbACQ, usbAMP ; Emka technologies SAS, Paris, France). End-Tidal CO2 (EtCO2) was measured using a probe placed distally to the pneumotachograph (Irma CO2 probe monnal, Masimo Corporation CA, USA). An esophageal balloon (MBMEd Prob. MBMED SA, Buenos Aires, Argentina) was inserted to measure esophageal pressure (Peso).

\section{Measured parameters.}

Haemodynamics. The following parameters were continuously monitored and recorded: Heart Rate (HR), Systolic Blood Pressure (SAP), Diastolic Blood Pressure (DAP), Carotid Blood Flow (CBF), Intra Cranial Pressure (ICP), Central Venous Pressure (CVP), Flow and Airway Pressure (Paw), Esophageal Pressure (Peso). During CPR, SAP was assumed to be the maximum of arterial pressure generated by the chest compression, and DAB to be the minimum pressure measured during decompression. Transpulmonary pressure (PL) was calculated as Paw minus Peso, cerebral perfusion pressure (CePP) was calculated as MAP minus ICP. Data were computed using a designated analysis program (IOX2 2.9.5.73®, EMKA Technologies, France). Cerebral and peripheral NIRS data were continuously monitored and recorded and extracted with the corresponding software.

Biology. Arterial blood gas, haemoglobin and lactate levels were assessed in an acid-base and co-oxymeter analyser at $38^{\circ} \mathrm{C}\left(\right.$ VetStat ${ }^{\mathrm{TM}}, \mathrm{IDEXX} \mathrm{Laboratories,}$ France). Lactate concentrations were determined using a Statstrip Lactate Xpress Meter (Nova Biomedical, Flintshire, UK). Plasma levels of troponin, brain natriuretic peptide (BNP), S100 calcium-binding protein B (S100B) and Neuron-specific Enolase (NSE) were measured using a standard chemistry analyser. The alveolar partial pressure of oxygen $\left(\mathrm{P}_{\mathrm{A}} \mathrm{O}_{2}\right)$ was calculated with the alveolar gas equation as $(16): \mathrm{P}_{\mathrm{A}} \mathrm{O}_{2}=\mathrm{PIO} 2-\mathrm{PaCO} / \mathrm{R}=(\mathrm{PB}-\mathrm{PH} \mathrm{O}) \star \mathrm{FIO} 2-(\mathrm{PaCO} / \mathrm{R})$ with $\mathrm{P}_{\mathrm{B}}$ : atmospheric pressure at sea level, $760 \mathrm{mmHg} ; \mathrm{P}_{\mathrm{H} 2 \mathrm{O}}$ : the saturated vapor pressure of water at body temperature and the prevailing atmospheric pressure, 47; FIO2: The fraction of inspired gas that is oxygen, $1 ; \mathrm{P}_{\mathrm{a}} \mathrm{CO}_{2}$ : The arterial partial pressure of carbon dioxide; $\mathrm{R}$ : The respiratory exchange ratio, 0.8

Histology. After sacrifice, autopsy was performed immediately with a sternotomy. From each lung, tissue sample from base, apex, anterior and posterior part were examined for injuries and atelectasis. All samples were initially immersion-fixed in $10 \%$ buffered formalin and subsequently imbedded in paraffin. Histopathological analysis was performed on $4 \mu \mathrm{m}$ tissue cut sections. The specimens were stained with haematoxylin-eosin. Macroscopy injury of the lung was evaluated with an anatomic scale of visual contusion (percentage of the whole lung). Lung tissue samples from each part were classified for intraalveolar haemorrhage, intra-alveolar oedema, atelectasia, alveolar distention and peri-alveolar bleeding as none ( $0 \%$ of the surface), light $(<25 \%$ of the surface), mild ( $25-75 \%$ of the surface), severe (> $75 \%$ of the surface).

\section{Experimental protocol.}

\section{Cardiac arrest model}

The overall protocol is shown in Fig. 1. The animal position on the table was secured with legs link. LUCA ${ }^{\text {TM }}$ device (Jolife AB/Physio-control, Lund, Sweden) was placed on the flat surface of the pig's thorax and securely positioned. Cardiac arrest was induced using a pacemaker wire inserted in the right ventricle through the venous catheter. Ventricular fibrillation was induced using a 9 Volt shock. The T0 time was defined as cardiac arrest. At T0, ventilation was stopped and a five minutes No-Flow period was respected. At T5 the animals were randomized according to the ventilation group and the LUCAS ${ }^{\text {TM }}$ device was started 100 per minute chest compressions (CC), without vasopressor administration during the protocol. Measurements occurred at Baseline Time (TB), Noflow beginning (T0), No-flow end/CPR starting (T5), and every five minutes (T10, T15, T20, T25, T30) until the end of protocol. Autopsy was then realized.

Groups. Animals were randomly assigned into three groups at time of LUCAS ${ }^{\mathrm{T}}$ initiation (T5): i) Standard Group (SD-group), ventilation according to the international recommendations (Monnal T60, Air Liquide Medical System, Antony, France) with the following settings : Volume control (VC), Vt 6 ml.kg-1, respiratory rate 10.minute ${ }^{-1}$, FiO2 100\%; ii) Cardio-Pulmonary-Ventilation Group (CPV-group), ventilation in pressure-controled CPV mode (CPV Monnal T60, Air Liquide Medical System, Antony, France) synchronized with CC, lower pressure at $5 \mathrm{cmH}_{2} \mathrm{O}$, upper pressure at $20 \mathrm{cmH}_{2} \mathrm{O}$, respiratory rate 10 .minute ${ }^{-1}$, FiO2 $100 \%$; iii) B-CARD group (BC-group), continuous oxygen insufflation (COI) with Boussignac Cardiac Arrest device (B-card, Vygon, Ecouen, France), 02 flow 15 L. $\min ^{-1}$ with no additional intervention. 


\section{Data analysis.}

For continuous data, recorded file using IOX (IOX2 2.9.5.73®, EMKA Technologies, France) were analysed with AcqKnowledge software (AcqKnowledge 3.7.3, Biopac Systems, Goleta, CA). Time periods were split into intervals: baseline to T0 (TB), T0 to T5 as No-Flow period (T5), T5 to T10 (T10), T10 to T15 (T15), T15 to T20 (T20), T20 to T25 (T25), T25 to T30 (T30).

\section{Statistics.}

Data are summarized as mean ( $\pm 1 \mathrm{SD}$ ) for continuous variables and count (\%) for categorical variables. Comparisons between the three groups for proportions were assessed with Fisher's exact test. Kruskal-Wallis one-way analysis of variance was used to compare continuous variables. The primary analysis compared both $\mathrm{PaCO} 2$ and $\mathrm{PaO} 2$ between the three groups. A regression model was fitted to analyze effects between groups. To account for the structure of the data, i.e. longitudinal data with repeated measures and a resulting intra-subject correlation, a logistic regression modelling was fitted using a generalized estimating equation method. Odds ratios (OR) were provided together with their $95 \%$ confidence intervals (95\% Cl). Secondary outcomes were investigated in a similar approach. When appropriate, analyses were performed after multiple imputations of missing data ("mitml” package for R). The statistical power was calculated posteriori. All statistical tests were two-sided and a p-value $\leq 0.05$ considered significant. Statistical analysis was performed with R software version 3.3.3 for Windows (R Foundation for Statistical Computing, Vienna, Austria). All figures were generated with Graph Pad Prism 8.0 software (GraphPad Software Inc, San Diego, CA)

\section{Results}

A total of 24 animals were studied: eight in SD-group, eight in CVP-group and eight in the BC-group. The three groups behavior were similar during baseline period [TB-TO] for all hemodynamic, ventilatory and biology parameters as well as during the No-Flow period [T0-T5] before randomization (Table 1). All animals had a Ventricular Fibrillation (VF) rhythm and only gasps were observed on airways probes. 
Table 1

Baseline parameters and at the end of five minutes of Cardiac arrest period (just before randomization).

\begin{tabular}{|c|c|c|c|c|c|c|}
\hline \multirow[t]{2}{*}{ Parameters } & \multicolumn{3}{|l|}{ Baseline } & \multicolumn{3}{|l|}{ T5 } \\
\hline & $S D(n=8)$ & $\mathrm{CPV}(\mathrm{n}=8)$ & $\mathrm{BC}(\mathrm{n}=8)$ & $\mathrm{SD}(\mathrm{n}=8)$ & $\mathrm{CPV}(\mathrm{n}=8)$ & $\mathrm{BC}(\mathrm{n}=8)$ \\
\hline weight (kg) & $50 \pm 10$ & $53 \pm 8$ & $45 \pm 5$ & & & \\
\hline \multicolumn{7}{|l|}{ Haemodynamic } \\
\hline $\mathrm{SAP}(\mathrm{mmHg})$ & $135 \pm 21$ & $134 \pm 26$ & $144 \pm 19$ & $34 \pm 9$ & $34 \pm 9$ & $28 \pm 6$ \\
\hline $\mathrm{DAP}(\mathrm{mmHg})$ & $88 \pm 22$ & $83 \pm 22$ & $95 \pm 18$ & $27 \pm 2$ & $29 \pm 7$ & $25 \pm 6$ \\
\hline MAP (mmHg) & $104 \pm 21$ & $101 \pm 23$ & $111 \pm 19$ & $29 \pm 3$ & $30 \pm 7$ & $26 \pm 6$ \\
\hline $\mathrm{CBF}\left(\mathrm{mL} \cdot \mathrm{min}^{-1}\right)$ & $243 \pm 85$ & $200 \pm 99$ & $191 \pm 91$ & $2.5 \pm 2.5$ & $2 \pm 1.2$ & $2 \pm 0.6$ \\
\hline CVP (mmHg) & $15 \pm 3$ & $14 \pm 3$ & $13 \pm 5$ & $25 \pm 5$ & $25 \pm 3$ & $24 \pm 5$ \\
\hline \multicolumn{7}{|l|}{ Cerebral and tissular } \\
\hline ICP (mmHg) & $14 \pm 4$ & $11 \pm 4$ & $14 \pm 6$ & $23 \pm 4$ & $20 \pm 5$ & $21 \pm 7$ \\
\hline $\mathrm{CePP}(\mathrm{mmHg})$ & $90 \pm 24$ & $91 \pm 25$ & $92 \pm 12$ & $6 \pm 5$ & $12 \pm 8$ & $6 \pm 8$ \\
\hline $\mathrm{c}-\mathrm{rSO} \mathrm{S}_{2}(\%)$ & $55 \pm 2$ & $56 \pm 5$ & $54 \pm 3$ & $41 \pm 3$ & $45 \pm 5$ & $43 \pm 6$ \\
\hline $\mathrm{t}-\mathrm{rSO}_{2}(\%)$ & $55 \pm 7$ & $54 \pm 9$ & $53 \pm 9$ & $24 \pm 6$ & $31 \pm 8$ & $22 \pm 9$ \\
\hline \multicolumn{7}{|l|}{ Ventilatory } \\
\hline Paw $\left(\mathrm{cmH}_{2} \mathrm{O}\right)$ & $10 \pm 5$ & $10 \pm 7$ & $10 \pm 5$ & $0 \pm 0$ & $0 \pm 0$ & $0 \pm 0$ \\
\hline Peso $\left(\mathrm{cmH}_{2} \mathrm{O}\right)$ & $28 \pm 19$ & $22 \pm 23$ & $24 \pm 22$ & $18 \pm 11$ & $17 \pm 15$ & $16 \pm 14$ \\
\hline Vte $\left(\mathrm{mL} \cdot \mathrm{kg}^{-1} \cdot \mathrm{min}^{-1}\right)$ & $9.3 \pm 1.8$ & $9.4 \pm 0.9$ & $9.9 \pm 1.5$ & $0 \pm 0$ & $0 \pm 0$ & $0 \pm 0$ \\
\hline Vti $\left(m L \cdot k g^{-1} \cdot \mathrm{min}^{-1}\right)$ & $9.8 \pm 1.2$ & $9.3 \pm 1.5$ & $9.9 \pm 1.6$ & $0 \pm 0$ & $0 \pm 0$ & $0 \pm 0$ \\
\hline $\mathrm{EtCO}_{2}(\mathrm{mmHg})$ & $40 \pm 0.6$ & $36 \pm 7$ & $44 \pm 3$ & $0 \pm 0$ & $0 \pm 0$ & $0 \pm 0$ \\
\hline \multicolumn{7}{|l|}{ Blood Gaz } \\
\hline $\mathrm{pH}$ & $7.4 \pm 0.1$ & $7.4 \pm 0.1$ & $7.4 \pm 0.1$ & $7.4 \pm 0.1$ & $7.4 \pm 0.1$ & $7.4 \pm 0.1$ \\
\hline $\mathrm{P}_{\mathrm{a}} \mathrm{CO} 2(\mathrm{mmHg})$ & $46 \pm 6$ & $45 \pm 5$ & $45 \pm 6$ & $47 \pm 10$ & $51 \pm 10$ & $54 \pm 17$ \\
\hline $\mathrm{P}_{\mathrm{a}} \mathrm{O} 2(\mathrm{mmHg})$ & $105 \pm 16$ & $106 \pm 20$ & $108 \pm 9$ & $74 \pm 33$ & $67 \pm 29$ & $70 \pm 29$ \\
\hline $\mathrm{P}_{\mathrm{A}} \mathrm{O} 2(\mathrm{mmHg})$ & $90 \pm 6$ & $93 \pm 6$ & $92 \pm 7$ & $90 \pm 14$ & $85 \pm 13$ & $81 \pm 22$ \\
\hline $\mathrm{HCO}_{3}^{-}\left(\mathrm{mmol} \cdot \mathrm{L}^{-1}\right)$ & $27 \pm 1$ & $26 \pm 1$ & $27 \pm 2$ & $27 \pm 2$ & $26 \pm 2$ & $27 \pm 3$ \\
\hline $\mathrm{Hb}\left(\mathrm{g} \cdot \mathrm{dL}^{-1}\right)$ & $9.6 \pm 2$ & $9.6 \pm 1$ & $9.1 \pm 1$ & $10.2 \pm 1$ & $10.7 \pm 2$ & $10.4 \pm 2$ \\
\hline $\mathrm{SpO} 2(\%)$ & $94 \pm 3$ & $94 \pm 2$ & $94 \pm 1$ & $84 \pm 15$ & $82 \pm 14$ & $82 \pm 14$ \\
\hline Lactate $\left(\mathrm{mmol} . \mathrm{L}^{-1}\right)$ & $1.5 \pm 0.5$ & $1.6 \pm 0.3$ & $1.5 \pm 0.3$ & $2 \pm 0.7$ & $2.5 \pm 1.2$ & $2.4 \pm 1.3$ \\
\hline \multicolumn{7}{|l|}{ Biology } \\
\hline Troponine $\left(\mu \mathrm{mol} . \mathrm{L}^{-1}\right)$ & $0.1 \pm 0.04$ & $0.1 \pm 0.01$ & $0.1 \pm 0.04$ & $87.8 \pm 27$ & $89.9 \pm 35$ & $134 \pm 70$ \\
\hline $\mathrm{BNP}\left(\mathrm{pg} \cdot \mathrm{mL}^{-1}\right)$ & $7.3 \pm 1.7$ & $6.7 \pm 1.2$ & $6.5 \pm 2$ & $36.8 \pm 9$ & $32.7 \pm 4$ & $32.6 \pm 5$ \\
\hline $\operatorname{NSE}\left(\mu \mathrm{mol} . \mathrm{L}^{-1}\right)$ & $1.6 \pm 0.2$ & $1.4 \pm 0.3$ & $1.5 \pm 0.2$ & $2.1 \pm 0.4$ & $1.8 \pm 0.5$ & $1.2 \pm 0.5$ \\
\hline S100B (pg.mL $\left.L^{-1}\right)$ & $0.4 \pm 0.01$ & $0.4 \pm 0.01$ & $0.4 \pm 0.1$ & $1.6 \pm 0.3$ & $1.6 \pm 0.2$ & $1.7 \pm 0.4$ \\
\hline
\end{tabular}

CA: cardiac arrest; SD: Standard ventilation Group; CPV: Cardio-Pulmonary Ventilation Group; BC: B-CARD Group; SAP: Systolic Arterial Pressure; DAP: Diastolic Arterial Pressure; MAP: Mean Arterial Pressure; CVP: Central Venous Pressure; ICP: Intra Cranial Pressure; CePP: Cerebral Perfusion Pressure; c-rSO 2 : regional saturation measured with Near Infra Red Spectroscopy; t-rSO ${ }_{2}$ : tissular Near Infra Red Spectroscopy; Paw: Airway Pressure; Peso: esophageal Pressure; Vte: Expriratory Tidal Volume; Vti: Inspiratory Tidal Volume; pH: Hydrogen Potential; pCO2: carbon dioxide partial pressure; p02: oxygen partial pressure; $\mathrm{HCO}_{3}{ }^{-}$: bicarbonate ; $\mathrm{Hb}$ : hemoglobin; SpO2: peripheral oxygen saturation; BNP: brain natriuretic peptide; NSE: Neuron-specific Enolase; S100B: S100 calcium-binding protein $\mathrm{B}$. Values are given as mean $\pm \mathrm{SD}$. 
Blood gases and ventilation measurements.

From T10 to T30, the evolution of pH was significantly different $(\mathrm{p}<0.05)$ as detailed in Table 2 . PaCO2 was significantly higher in the BC-group $(>90 \mathrm{mmHg})$ with a positive effect of time ( $<<0.001$; Table ESM 1.1); PaO2 was significantly higher in the SD group (>200 mmHg) (Table 2, Fig. 1) with no effect of time ( $p$ = 0.69; Table ESM 1.2). The tidal volume (Vti) delivered was significantly dependent of the device tested ( $<<0.001)$, with higher volume for SD-group, as well as higher Paw and intra-thoracic pressure. (Table 3).

Table 2

Blood gas parameters evolution after randomization

\begin{tabular}{|c|c|c|c|c|c|c|c|c|c|c|}
\hline \multirow[t]{2}{*}{ Parameters } & \multicolumn{3}{|l|}{$\mathrm{T} 10$} & \multicolumn{3}{|l|}{ T20 } & \multicolumn{4}{|l|}{ T30 } \\
\hline & $\begin{array}{l}S D(n= \\
8)\end{array}$ & $\begin{array}{l}\text { CPV }(n \\
=8)\end{array}$ & $\begin{array}{l}B C(n= \\
8)\end{array}$ & $\begin{array}{l}S D(n \\
=8)\end{array}$ & $\begin{array}{l}\text { CPV }(n \\
=8)\end{array}$ & $\begin{array}{l}B C(n= \\
8)\end{array}$ & $\begin{array}{l}S D(n \\
=8)\end{array}$ & $\begin{array}{l}\text { CPV }(n \\
=8)\end{array}$ & $\begin{array}{l}\mathrm{BC}(\mathrm{n}= \\
8)\end{array}$ & $\begin{array}{l}\mathrm{p} \text { (Kurskal- } \\
\text { Wallis) }\end{array}$ \\
\hline \multicolumn{11}{|l|}{ Blood Gaz } \\
\hline $\mathrm{pH}$ & $\begin{array}{l}7.32 \pm \\
0.11\end{array}$ & $\begin{array}{l}7.21 \pm \\
0.07\end{array}$ & $\begin{array}{l}7.11 \pm \\
0.06\end{array}$ & $\begin{array}{l}7.25 \pm \\
0.1\end{array}$ & $\begin{array}{l}7.15 \pm \\
0.11\end{array}$ & $\begin{array}{l}6.98 \pm \\
0.06\end{array}$ & $\begin{array}{l}7.19 \pm \\
0.14\end{array}$ & $\begin{array}{l}7.05 \pm \\
0.14\end{array}$ & $\begin{array}{l}6.89 \pm \\
0.10\end{array}$ & $<0.01$ * \\
\hline $\begin{array}{l}\mathrm{P}_{\mathrm{a}} \mathrm{CO} 2 \\
(\mathrm{mmHg})\end{array}$ & $\begin{array}{l}45.5 \pm \\
10.3\end{array}$ & $\begin{array}{l}68.9 \pm \\
15.4\end{array}$ & $\begin{array}{l}94.3 \pm \\
17.2\end{array}$ & $\begin{array}{l}45 \pm \\
9.4\end{array}$ & $\begin{array}{l}65.4 \pm \\
22.7\end{array}$ & $\begin{array}{l}120.1 \pm \\
21.2\end{array}$ & $\begin{array}{l}49.8 \pm \\
22.1\end{array}$ & $\begin{array}{l}78 \pm \\
25.1\end{array}$ & $\begin{array}{l}146.3 \pm \\
45\end{array}$ & $<0.01$ * \\
\hline $\mathrm{P}_{\mathrm{a}} \mathrm{O} 2(\mathrm{mmHg})$ & $\begin{array}{l}263 \pm \\
105\end{array}$ & $\begin{array}{l}118 \pm \\
50\end{array}$ & $96 \pm 51$ & $\begin{array}{l}336 \pm \\
136\end{array}$ & $\begin{array}{l}131 \pm \\
84\end{array}$ & $96 \pm 52$ & $\begin{array}{l}216 \pm \\
147\end{array}$ & $76 \pm 31$ & $68 \pm 44$ & $<0.01$ * \\
\hline $\mathrm{P}_{\mathrm{A}} \mathrm{O}_{2}(\mathrm{mmHg})$ & $\begin{array}{l}656 \pm \\
13\end{array}$ & $\begin{array}{l}626 \pm \\
19\end{array}$ & $\begin{array}{l}595 \pm \\
21\end{array}$ & $\begin{array}{l}657 \pm \\
12\end{array}$ & $\begin{array}{l}631 \pm \\
28\end{array}$ & $563 \pm 26$ & $\begin{array}{l}651 \pm \\
28\end{array}$ & $\begin{array}{l}616 \pm \\
31\end{array}$ & $\begin{array}{l}530 \pm \\
56\end{array}$ & 0.21 \\
\hline $\begin{array}{l}\mathrm{HCO}_{3}^{-} \\
\left(\mathrm{mmol} . \mathrm{L}^{-1}\right)\end{array}$ & $\begin{array}{l}20.8 \pm \\
1.8\end{array}$ & $\begin{array}{l}23.8 \pm \\
2.7\end{array}$ & $\begin{array}{l}27.2 \pm \\
3.3\end{array}$ & $\begin{array}{l}17.8 \pm \\
1.6\end{array}$ & $\begin{array}{l}19.5 \pm \\
2.9\end{array}$ & $\begin{array}{l}24.9 \pm \\
2.7\end{array}$ & $\begin{array}{l}16 \pm \\
2.2\end{array}$ & $\begin{array}{l}18.6 \pm \\
2.2\end{array}$ & $\begin{array}{l}24.1 \pm \\
4.4\end{array}$ & $<0.01$ * \\
\hline $\mathrm{Hb}\left(\mathrm{g} \cdot \mathrm{dL}^{-1}\right)$ & $\begin{array}{l}11.7 \pm \\
1.5\end{array}$ & $\begin{array}{l}12.3 \pm \\
0.9\end{array}$ & $\begin{array}{l}11.9 \pm \\
1.6\end{array}$ & $\begin{array}{l}11.4 \pm \\
1.6\end{array}$ & $\begin{array}{l}12.2 \pm \\
0.9\end{array}$ & $\begin{array}{l}12.1 \pm \\
1.8\end{array}$ & $\begin{array}{l}11.6 \pm \\
1.9\end{array}$ & $\begin{array}{l}12.7 \pm \\
1.1\end{array}$ & $\begin{array}{l}12.4 \pm \\
2.4\end{array}$ & $<0.01 \#$ \\
\hline SpO2 (\%) & $99 \pm 3$ & $91 \pm 7$ & $82 \pm 15$ & $99 \pm 1$ & $90 \pm 7$ & $79 \pm 17$ & $89 \pm 18$ & $77 \pm 14$ & $70 \pm 14$ & $<0.01$ * \\
\hline $\begin{array}{l}\text { Lactate } \\
\left(\mathrm{mmol} . \mathrm{L}^{-1}\right)\end{array}$ & & & & & & & $\begin{array}{l}7.5 \pm \\
1.2\end{array}$ & $\begin{array}{l}8.5 \pm \\
0.9\end{array}$ & $\begin{array}{l}8.2 \pm \\
1.7\end{array}$ & 0.83 \\
\hline
\end{tabular}

SD: Standard ventilation Group; CPV: Cardio-Pulmonary Ventilation Group; BC: B-CARD Group; pH: Hydrogen Potential; pCO2: carbon dioxide partial pressure; p02: oxygen partial pressure; $\mathrm{HCO}_{3}$ : bicarbonate; $\mathrm{Hb}$ : hemoglobin; $\mathrm{SpO}$ : peripheral oxygen saturation. Values are given as mean $\pm \mathrm{SD}$. Kruskal-Wallis oneway analysis of variance was used to compare continuous variables. The sign displays the bonferroni corrected p-values from pairwise comparisons between groups performed by the Dunn's post hoc test and Dunn's post-hoc test was used to compare groups: \# SD vs. CPV, * SD vs. BC, § CPV vs. BC 
Table 3

Haemodynamic, cerebral, tissular, ventilatory parameters evolution after randomization.

\begin{tabular}{|c|c|c|c|c|c|c|c|c|c|c|c|c|c|c|c|}
\hline Parameters & T10 & & & $\mathrm{T} 15$ & & & T20 & & & T25 & & & T30 & & \\
\hline & $\begin{array}{l}\text { SD } \\
(n= \\
8)\end{array}$ & $\begin{array}{l}\text { CPV }(n \\
=8)\end{array}$ & $\begin{array}{l}\mathrm{BC} \\
(\mathrm{n} \\
=8)\end{array}$ & $\begin{array}{l}S D \\
(n= \\
8)\end{array}$ & $\begin{array}{l}\text { CPV } \\
(n= \\
8)\end{array}$ & $\begin{array}{l}\text { BC } \\
(n \\
= \\
8)\end{array}$ & $\begin{array}{l}\text { SD } \\
(n= \\
8)\end{array}$ & $\begin{array}{l}C P V \\
(n= \\
8)\end{array}$ & $\begin{array}{l}B C \\
(n= \\
8)\end{array}$ & $\begin{array}{l}S D \\
(n= \\
8)\end{array}$ & $\begin{array}{l}\text { CPV } \\
(n= \\
8)\end{array}$ & $\begin{array}{l}\mathrm{BC} \\
(\mathrm{n} \\
= \\
8)\end{array}$ & $\begin{array}{l}\text { SD } \\
(n= \\
8)\end{array}$ & $\begin{array}{l}C P V \\
(n= \\
8)\end{array}$ & $\begin{array}{l}\mathrm{BC} \\
(\mathrm{n} \\
= \\
8)\end{array}$ \\
\hline \multicolumn{16}{|l|}{ Haemodynamic } \\
\hline SAP (mmHg) & $\begin{array}{l}107 \\
\pm 35\end{array}$ & $\begin{array}{l}79 \pm \\
17\end{array}$ & $\begin{array}{l}90 \\
\pm \\
40\end{array}$ & $\begin{array}{l}85 \pm \\
39\end{array}$ & $\begin{array}{l}73 \\
\pm \\
28\end{array}$ & $\begin{array}{l}67 \\
\pm \\
30\end{array}$ & $\begin{array}{l}71 \pm \\
29\end{array}$ & $\begin{array}{l}61 \\
\pm \\
18\end{array}$ & $\begin{array}{l}58 \pm \\
33\end{array}$ & $\begin{array}{l}66 \pm \\
34\end{array}$ & $\begin{array}{l}50 \\
\pm \\
15\end{array}$ & $\begin{array}{l}43 \\
\pm \\
23\end{array}$ & $\begin{array}{l}56 \pm \\
33\end{array}$ & $\begin{array}{l}38 \\
\pm \\
10\end{array}$ & $\begin{array}{l}33 \\
\pm \\
26\end{array}$ \\
\hline DAP (mmHg) & $\begin{array}{l}36 \pm \\
31\end{array}$ & $25 \pm 5$ & $\begin{array}{l}27 \\
\pm \\
12\end{array}$ & $\begin{array}{l}22 \pm \\
5\end{array}$ & $\begin{array}{l}30 \\
\pm \\
16\end{array}$ & $\begin{array}{l}23 \\
\pm 9\end{array}$ & $22 \pm$ & $\begin{array}{l}22 \\
\pm 4\end{array}$ & $\begin{array}{l}22 \pm \\
9\end{array}$ & $\begin{array}{l}21 \pm \\
10\end{array}$ & $\begin{array}{l}20 \\
\pm 5\end{array}$ & $\begin{array}{l}20 \\
\pm 9\end{array}$ & $\begin{array}{l}18 \pm \\
8\end{array}$ & $\begin{array}{l}16 \\
\pm 3\end{array}$ & $\begin{array}{l}18 \\
\pm \\
12\end{array}$ \\
\hline MAP (mmHg) & $\begin{array}{l}60 \pm \\
28\end{array}$ & $43 \pm 7$ & $\begin{array}{l}48 \\
\pm \\
21\end{array}$ & $\begin{array}{l}43 \pm \\
15\end{array}$ & $\begin{array}{l}45 \\
\pm \\
19\end{array}$ & $\begin{array}{l}38 \\
\pm \\
15\end{array}$ & $\begin{array}{l}39 \pm \\
14\end{array}$ & $\begin{array}{l}35 \\
\pm 7\end{array}$ & $\begin{array}{l}34 \pm \\
17\end{array}$ & $\begin{array}{l}36 \pm \\
18\end{array}$ & $\begin{array}{l}30 \\
\pm 7\end{array}$ & $\begin{array}{l}28 \\
\pm \\
14\end{array}$ & $\begin{array}{l}31 \pm \\
16\end{array}$ & $\begin{array}{l}24 \\
\pm 5\end{array}$ & $\begin{array}{l}23 \\
\pm \\
17\end{array}$ \\
\hline $\mathrm{CBF}\left(\mathrm{ml} \cdot \mathrm{min}^{-1}\right)$ & $\begin{array}{l}80 \pm \\
11\end{array}$ & $\begin{array}{l}110 \pm \\
159\end{array}$ & $\begin{array}{l}98 \\
\pm \\
118\end{array}$ & $\begin{array}{l}68 \pm \\
38\end{array}$ & $\begin{array}{l}67 \\
\pm \\
38\end{array}$ & $\begin{array}{l}47 \\
\pm \\
51\end{array}$ & $\begin{array}{l}66 \pm \\
25\end{array}$ & $\begin{array}{l}49 \\
\pm \\
21\end{array}$ & $\begin{array}{l}37 \pm \\
28\end{array}$ & $\begin{array}{l}56 \pm \\
26\end{array}$ & $\begin{array}{l}36 \\
\pm \\
26\end{array}$ & $\begin{array}{l}40 \\
\pm \\
44\end{array}$ & $\begin{array}{l}45 \pm \\
28\end{array}$ & $\begin{array}{l}17 \\
\pm \\
25\end{array}$ & $\begin{array}{l}17 \\
\pm \\
19\end{array}$ \\
\hline CVP (mmHg) & $\begin{array}{l}38 \pm \\
18\end{array}$ & $\begin{array}{l}40 \pm \\
31\end{array}$ & $\begin{array}{l}38 \\
\pm \\
17\end{array}$ & $\begin{array}{l}33 \pm \\
18\end{array}$ & $\begin{array}{l}29 \\
\pm \\
17\end{array}$ & $\begin{array}{l}30 \\
\pm 9\end{array}$ & $\begin{array}{l}29 \pm \\
14\end{array}$ & $\begin{array}{l}27 \\
\pm \\
16\end{array}$ & $\begin{array}{l}28 \pm \\
10\end{array}$ & $\begin{array}{l}28 \pm \\
13\end{array}$ & $\begin{array}{l}25 \\
\pm \\
15\end{array}$ & $\begin{array}{l}26 \\
\pm 9\end{array}$ & $\begin{array}{l}27 \pm \\
11\end{array}$ & $\begin{array}{l}25 \\
\pm \\
14\end{array}$ & $\begin{array}{l}26 \\
\pm 9\end{array}$ \\
\hline \multicolumn{16}{|l|}{$\begin{array}{l}\text { Cerebral and } \\
\text { tissular }\end{array}$} \\
\hline ICP (mmHg) & $\begin{array}{l}25 \pm \\
6\end{array}$ & $\begin{array}{l}22 \pm \\
11\end{array}$ & $\begin{array}{l}20 \\
\pm 7\end{array}$ & $\begin{array}{l}23 \pm \\
6\end{array}$ & $\begin{array}{l}16 \\
\pm 5\end{array}$ & $\begin{array}{l}17 \\
\pm 9\end{array}$ & $21 \pm$ & $\begin{array}{l}14 \\
\pm 5\end{array}$ & $\begin{array}{l}16 \pm \\
9\end{array}$ & $\begin{array}{l}21 \pm \\
7\end{array}$ & $\begin{array}{l}14 \\
\pm 5\end{array}$ & $\begin{array}{l}16 \\
\pm 9\end{array}$ & $\begin{array}{l}20 \pm \\
7\end{array}$ & $\begin{array}{l}13 \\
\pm 5\end{array}$ & $\begin{array}{l}16 \\
\pm 8\end{array}$ \\
\hline CePP $(\mathrm{mmHg})$ & $\begin{array}{l}24 \pm \\
18\end{array}$ & $26 \pm 7$ & $\begin{array}{l}27 \\
\pm \\
23\end{array}$ & $\begin{array}{l}19 \pm \\
19\end{array}$ & $\begin{array}{l}31 \\
\pm \\
16\end{array}$ & $\begin{array}{l}22 \\
\pm \\
18\end{array}$ & $\begin{array}{l}17 \pm \\
18\end{array}$ & $\begin{array}{l}22 \\
\pm 8\end{array}$ & $\begin{array}{l}21 \pm \\
19\end{array}$ & $\begin{array}{l}15 \pm \\
23\end{array}$ & $\begin{array}{l}16 \\
\pm 8\end{array}$ & $\begin{array}{l}15 \\
\pm \\
15\end{array}$ & $\begin{array}{l}11 \pm \\
19\end{array}$ & $\begin{array}{l}10 \\
\pm 7\end{array}$ & $\begin{array}{l}11 \\
\pm \\
19\end{array}$ \\
\hline $\mathrm{c}-\mathrm{rSO}_{2}(\%)$ & $\begin{array}{l}40 \pm \\
3\end{array}$ & $41 \pm 7$ & $\begin{array}{l}41 \\
\pm 7\end{array}$ & $\begin{array}{l}41 \pm \\
4\end{array}$ & $\begin{array}{l}43 \\
\pm 5\end{array}$ & $\begin{array}{l}42 \\
\pm 6\end{array}$ & $\begin{array}{l}41 \pm \\
4\end{array}$ & $\begin{array}{l}42 \\
\pm 6\end{array}$ & $\begin{array}{l}42 \pm \\
6\end{array}$ & $\begin{array}{l}40 \pm \\
5\end{array}$ & $\begin{array}{l}41 \\
\pm 7\end{array}$ & $\begin{array}{l}41 \\
\pm 7\end{array}$ & $\begin{array}{l}39 \pm \\
5\end{array}$ & $\begin{array}{l}41 \\
\pm 7\end{array}$ & $\begin{array}{l}41 \\
\pm 7\end{array}$ \\
\hline $\mathrm{t}-\mathrm{rSO} \mathrm{S}_{2}(\%)$ & $\begin{array}{l}23 \pm \\
9\end{array}$ & $25 \pm 6$ & $\begin{array}{l}17 \\
\pm \\
10\end{array}$ & $\begin{array}{l}37 \pm \\
4\end{array}$ & $\begin{array}{l}37 \\
\pm 2\end{array}$ & $\begin{array}{l}36 \\
\pm 4\end{array}$ & $\begin{array}{l}36 \pm \\
5\end{array}$ & $\begin{array}{l}36 \\
\pm 3\end{array}$ & $\begin{array}{l}33 \pm \\
5\end{array}$ & $\begin{array}{l}30 \pm \\
11\end{array}$ & $\begin{array}{l}29 \\
\pm \\
10\end{array}$ & $\begin{array}{l}21 \\
\pm \\
10\end{array}$ & $\begin{array}{l}26 \pm \\
14\end{array}$ & $\begin{array}{l}24 \\
\pm \\
10\end{array}$ & $\begin{array}{l}17 \\
\pm \\
10\end{array}$ \\
\hline \multicolumn{16}{|l|}{ Ventilatory } \\
\hline $\mathrm{EtCO} 2(\mathrm{mmHg})$ & $\begin{array}{l}25 \pm \\
11\end{array}$ & $\begin{array}{l}24 \pm \\
12\end{array}$ & $\begin{array}{l}28 \\
\pm 6\end{array}$ & $\begin{array}{l}32 \pm \\
11\end{array}$ & $\begin{array}{l}56 \\
\pm \\
36\end{array}$ & $\begin{array}{l}50 \\
\pm \\
33\end{array}$ & $\begin{array}{l}27 \pm \\
8\end{array}$ & $\begin{array}{l}59 \\
\pm \\
35\end{array}$ & $\begin{array}{l}52 \pm \\
33\end{array}$ & $\begin{array}{l}18 \pm \\
3\end{array}$ & $\begin{array}{l}57 \\
\pm \\
36\end{array}$ & $\begin{array}{l}49 \\
\pm \\
29\end{array}$ & $\begin{array}{l}13 \pm \\
5\end{array}$ & $\begin{array}{l}48 \\
\pm \\
28\end{array}$ & $\begin{array}{l}40 \\
\pm \\
23\end{array}$ \\
\hline Paw (cH2O) & $\begin{array}{l}16 \pm \\
11\end{array}$ & $12 \pm 6$ & $\begin{array}{l}6 \pm \\
4\end{array}$ & $\begin{array}{l}15 \pm \\
10\end{array}$ & $\begin{array}{l}12 \\
\pm 6\end{array}$ & $\begin{array}{l}7 \pm \\
4\end{array}$ & $\begin{array}{l}15 \pm \\
10\end{array}$ & $\begin{array}{l}12 \\
\pm 6\end{array}$ & $\begin{array}{l}6 \pm \\
3\end{array}$ & $\begin{array}{l}15 \pm \\
11\end{array}$ & $\begin{array}{l}12 \\
\pm 6\end{array}$ & $\begin{array}{l}6 \pm \\
3\end{array}$ & $\begin{array}{l}15 \pm \\
10\end{array}$ & $\begin{array}{l}12 \\
\pm 5\end{array}$ & $\begin{array}{l}6 \pm \\
3\end{array}$ \\
\hline Peso (cH2O) & $\begin{array}{l}37 \pm \\
22\end{array}$ & $\begin{array}{l}26 \pm \\
28\end{array}$ & $\begin{array}{l}27 \\
\pm \\
24\end{array}$ & $\begin{array}{l}32 \pm \\
21\end{array}$ & $\begin{array}{l}23 \\
\pm \\
25\end{array}$ & $\begin{array}{l}23 \\
\pm \\
20\end{array}$ & $\begin{array}{l}31 \pm \\
20\end{array}$ & $\begin{array}{l}21 \\
\pm \\
22\end{array}$ & $\begin{array}{l}22 \pm \\
18\end{array}$ & $\begin{array}{l}31 \pm \\
21\end{array}$ & $\begin{array}{l}21 \\
\pm \\
22\end{array}$ & $\begin{array}{l}22 \\
\pm \\
17\end{array}$ & $\begin{array}{l}31 \pm \\
22\end{array}$ & $\begin{array}{l}21 \\
\pm \\
22\end{array}$ & $\begin{array}{l}22 \\
\pm \\
17\end{array}$ \\
\hline $\mathrm{PL}(\mathrm{cH} 20)$ & $\begin{array}{l}-20 \\
\pm 18\end{array}$ & $\begin{array}{l}-15 \pm \\
22\end{array}$ & $\begin{array}{l}-21 \\
\pm \\
20\end{array}$ & $\begin{array}{l}-16 \\
\pm 16\end{array}$ & $\begin{array}{l}-11 \\
\pm \\
19\end{array}$ & $\begin{array}{l}-17 \\
\pm \\
17\end{array}$ & $\begin{array}{l}-15 \\
\pm 15\end{array}$ & $\begin{array}{l}-10 \\
\pm \\
17\end{array}$ & $\begin{array}{l}-16 \\
\pm 15\end{array}$ & $\begin{array}{l}-15 \\
\pm 16\end{array}$ & $\begin{array}{l}-9 \pm \\
17\end{array}$ & $\begin{array}{l}-16 \\
\pm \\
14\end{array}$ & $\begin{array}{l}-15 \\
\pm 17\end{array}$ & $\begin{array}{l}-9 \pm \\
16\end{array}$ & $\begin{array}{l}-16 \\
\pm \\
14\end{array}$ \\
\hline $\begin{array}{l}\text { Vti }\left(\mathrm{mL} \cdot \mathrm{kg}^{-}\right. \\
\left.{ }^{1} \cdot \mathrm{min}^{-1}\right)\end{array}$ & $\begin{array}{l}11.4 \\
\pm \\
1.6\end{array}$ & $\begin{array}{l}5.5 \pm \\
1.5\end{array}$ & $\begin{array}{l}4.5 \\
\pm \\
1.7\end{array}$ & $\begin{array}{l}11.4 \\
\pm \\
1.4\end{array}$ & $\begin{array}{l}5.4 \\
\pm \\
1.8\end{array}$ & $\begin{array}{l}4.3 \\
\pm \\
1.7\end{array}$ & $\begin{array}{l}11.3 \\
\pm \\
1.3\end{array}$ & $\begin{array}{l}5.4 \\
\pm \\
1.8\end{array}$ & $\begin{array}{l}4.4 \pm \\
1.8\end{array}$ & $\begin{array}{l}11.3 \\
\pm 2\end{array}$ & $\begin{array}{l}5.4 \\
\pm \\
1.8\end{array}$ & $\begin{array}{l}4.3 \\
\pm \\
1.8\end{array}$ & $\begin{array}{l}11.4 \\
\pm 2\end{array}$ & $\begin{array}{l}5.6 \\
\pm \\
2.1\end{array}$ & $\begin{array}{l}4.3 \\
\pm 2\end{array}$ \\
\hline $\begin{array}{l}\text { Vte }\left(\mathrm{mL} . \mathrm{kg}^{-}\right. \\
\left.{ }^{1} \cdot \mathrm{min}^{-1}\right)\end{array}$ & $\begin{array}{l}7.1 \\
\pm \\
4.1\end{array}$ & $\begin{array}{l}4.8 \pm \\
1.4\end{array}$ & $\begin{array}{l}4 \pm \\
1.6\end{array}$ & $\begin{array}{l}7.5 \\
\pm \\
3.9\end{array}$ & $\begin{array}{l}4.8 \\
\pm \\
1.4\end{array}$ & $\begin{array}{l}3.9 \\
\pm \\
1.5\end{array}$ & $\begin{array}{l}7.1 \\
\pm \\
3.8\end{array}$ & $\begin{array}{l}5.1 \\
\pm 1\end{array}$ & $\begin{array}{l}3.8 \\
\pm \\
1.6\end{array}$ & $\begin{array}{l}8.5 \\
\pm \\
5.2\end{array}$ & $\begin{array}{l}5.3 \\
\pm \\
1.3\end{array}$ & $\begin{array}{l}3.9 \\
\pm \\
1.6\end{array}$ & $\begin{array}{l}7.9 \\
\pm \\
3.8\end{array}$ & $\begin{array}{l}5.3 \\
\pm \\
1.3\end{array}$ & $\begin{array}{l}3.9 \\
\pm 2\end{array}$ \\
\hline
\end{tabular}

SD: Standard ventilation Group; CPV: Cardio-Pulmonary Ventilation Group; BC: B-CARD Group; SAP: Systolic Arterial Pressure; DAP: Diastolic Arterial Pressure; MAP: Mean Arterial Pressure; CVP: Central Venous Pressure; ICP: Intra Cranial Pressure; CePP: Cerebral Perfusion Pressure; c-rSO ${ }_{2}$ : regional saturation measured with Near Infra Red Spectroscopy; t-rSO ${ }_{2}$ : tissular Near Infra Red Spectroscopy; Paw: Airway Pressure; Peso: esophageal Pressure; Vte: Expriratory Tidal Volume; Vti: Inspiratory Tidal Volume. Values are given as mean \pm SD. Kruskal-Wallis one-way analysis of variance was used to compare continuous variables. The sign displays the bonferroni corrected p-values from pairwise comparisons between groups performed by the Dunn's post hoc test: \# SD vs. $\mathrm{CPV}$, * SD vs. BC, § CPV vs. BC.

Hemodynamics, cerebral perfusion and injury parameters during CPR 
MAP, CVP, $\mathrm{CBF}, \mathrm{t}-\mathrm{rSO}_{2}$, lactate evolution were not impacted by the ventilator device strategy (Table 3, Fig. 2.A), BNP serum level (pg.mL $\left.{ }^{-} 1\right)$ was not affected by the ventilatory mode (SD vs. CPV vs. BC) at the end of protocol $(37 \pm 10$ vs. $31 \pm 6$ vs.30 $\pm 6 ; \mathrm{p}=0.43)$. There was no ventilatory impact on CePP and c-rSO 2 (Table 3, Fig. 2.B). Cerebral parenchyma injury did not revealed differences between groups (SD vs. CPV vs. BC) assessed at the end of protocol by NSE ( \pm \pm 0.1 vs. $2 \pm 0.2$ vs. $\left.2 \pm 1 ; \mu \mathrm{mol} . \mathrm{L}^{-1} ; p=0.43\right)$ and SB100 (1.4 \pm 0.4 vs. $1.4 \pm 0.2$ vs. $\left.1.7 \pm 0.4 ; p=0.43\right)$

\section{Histopathological changes.}

Macroscopic estimation of the lung infarction was not different between the three groups $(p=0.09)$. The microscopic analysis of alveolar collapse, alveolar distension, intra-alveolar haemorrhage and peri-bronchiolar haemorrhage, are illustrated in Fig. 4 with the histopathological changes.

\section{Discussion}

The main results of our study are that (i) standard ventilation at FiO2 100\% was associated with major hyperoxia and efficient CO2 removal; (ii) CPV was associated with normoxia and efficient $\mathrm{CO} 2$ removal; (iii) B-CARD was associated with normoxia and major hypercapnia. Despite these differences, the ventilatory strategy did not impact hemodynamic and cerebral parameters.

\section{Hyperoxia and hypercapnia issue}

Hyperoxia is still an issue leading to significant neurological damage $(17,18)$. If the question is now well focused on the oxygenation target of patients in post-cardiac arrest (avoid hyperoxia as much as possible), the target during cardiopulmonary resuscitation remains unknown (19-21) and will require further clinical investigations (20). The higher Vt measured in SD group during the experiment could explain this major difference in oxygenation between group.

We observe a significant difference in arterial level of $\mathrm{CO} 2$ depending on the ventilation method used. Hypercapnia (PaCO2 > $45 \mathrm{mmHg})$ directly disables the organism's acid-base balance and thus has a direct impact on all organ's performances. While we were able to identify a very moderate increase in PaCO2 in the SD group, we found a significant increase in PaCO2 in the B-Card group and no major hypercapnia in the CPV group. This hypercapnia could be explained, based on the $\Delta \mathrm{Vti}-\mathrm{Vte}$ measurement, by trapping of the inspired volume with no exhaling during the compression phase. A randomized trial (NCT03347175) comparing three ventilation mode (volume controlled, BIPAP and CPAP) during CPR with MCC in emergency room will focus specifically on the question of volume inspired and consequences on gas exchanges. However, Schneider et al. were able to determine that, compared to normocapnia, hypercapnia was associated with a higher probability of survival regardless of the patient's neurological state (22). In addition, Eastwood et al. in a multicenter prospective study highlighted that moderate hypercapnia ( $\mathrm{PaCO} 250-55 \mathrm{mmHg}$ ) compared to normocapnia (PaCO2 $35-45 \mathrm{mmHg}$ ) induced a decrease in brain injury biomarker (NSE) production (23). Other trials are in progress (NCT03114033) assessing this therapeutic target. In our study, brain biomarkers trends were not correlated with PaCO2 level after 30 min of CPR.

\section{Hemodynamic issue}

We did not find any impact on hemodynamic parameters (no difference on MAP, CVP) or on brain circulation regardless of the ventilatory method used (no difference on CePP, NIRS) despite the major variations in $\mathrm{PaO} 2$ and $\mathrm{PaCO} 2$. In parallel, hyperoxia can have a direct vasoconstrictor effect, which can lead to a reduction in cerebral blood flow, thus increasing cerebral ischemia (24). In our model, CPV mode seemed to allow acceptable oxygenation and decarboxylation without hemodynamic impairment. Low MAP level did not allow us to explore the question of cerebral autoregulation (25), neither the relationship between NIRS and cerebral blood flow or CVP (26).

One key point of the influence of ventilation on hemodynamics is the impact of PEEP level during CPR (3). This subject is still controversial; for some authors applying PEEP could impact venous return by increasing intrathoracic pressure and have a negative impact on circulation (27); for others applying PEEP could enhance the airway opening and increase blood flow $(28,29)$. In our study we did not observe any impact on the right heart of PEEP level in CPV $(P E E P 5$ $\mathrm{cmH}_{2} \mathrm{O}$ ) nor B-Card (PEEP $5 \mathrm{cmH}_{2} \mathrm{O}$ ) strategy compared to Standard-group (PEEP $0 \mathrm{cmH}_{2} \mathrm{O}$ ), and no macro-hemodynamic impairment.

\section{Limitations}

However, our study has several limitations. First of all, we decided not to use catecholamines during resuscitation in order to avoid their effect on gas exchanges, acid/basic balance and hemodynamics. We chose to stop CPR at the end of the experiment with no ROSC goal because we didn't have the foresight to assess the animal's prognosis. Secondly, B-CARD strategy tested as permissive hypercapnia was probably put in difficulty with an increased experimental dead space due to the sensors necessary to the experiment. Nevertheless, the major hypercapnia observed did not influence hemodynamic parameters or cerebral perfusion. Thirdly, we did not observe any intrathoracic airway closure in our model (30) suggesting that porcine and human respiratory systems may behave differently. Indeed, the rather "peak" shape of the pig chest can also lead to some differences in analysis regarding gas exchanges and the effectiveness of active compression decompression. It would be interesting to be able to assess our hypotheses on revitalized cadavers in order to confirm the reproducibility of our results. Fourthly, choice was made to involve as few animals as possible to achieve research aims because of ethical concerns.

\section{Conclusion}

Ventilation modalities in this porcine model of prolonged CPR influenced oxygenation and decarboxylation without impairing circulation and cerebral perfusion. 


\section{Abbreviations}

BC-group

Boussignac Cardiac-Arrest Device

BNP

brain natriuretic peptide

CBF

Carotid Blood Flow

CCC

continuous chest compressions

CePP

cerebral perfusion pressure

cOI

continuous oxygen insufflation

CPR

cardio-pulmonary resuscitation

CPV-group

bi-level pressure mode

CVP

Central Venous Pressure

DAP

Diastolic Blood Pressure

EtCO2

End-Tidal $\mathrm{CO} 2$

HR

Heart Rate

ICP

Intra Cranial Pressure

MCCC

mechanical continuous chest compressions

NIRS

Near Infra Red Spectroscopy

NSE

Neuron-specific Enolase

$\mathrm{P}_{\mathrm{A}} \mathrm{O}_{2}$

alveolar partial pressure of oxygen

$\mathrm{PL}$

Transpulmonary pressure

PEEP

positive end-expiratory pressure

Paw

Airway Pressure

Peso

esophageal pressure

rSO2

regional saturation

SBP

Systolic Blood Pressure

S100B

S100 calcium-binding protein B

SD-group

Standard volume-control mode

\section{Declarations}

Ethical approval. All experiments were reviewed and approved by the Nancy University Ethics Committee for Animal Experimentation (APAFIS number 101992017061212039981v1). The procedure for the care and sacrifices of study animals was in accordance with the European Community Standards on the Care and Use of Laboratory Animals.

Consent for publication. Not applicable 
Availability of data and material. The datasets used and analysed during the current study are available from the corresponding autor on reasonable request.

Competing interests. JCM Richard receives a part time salary from Air Liquide Medical Systems (Antony, France). B. Badat and M. Rigollot are medical engineers employed by the society Air Liquide Medical Systems (Antony France). The other authors have no personal conflict of interest to report regarding to this experiment

Funding. This work was supported by the Lorraine University, Lorraine Association of Emergency Physicians. A Lucas II was lended by Medtronic (Mississauga, Canada). NIRS device was lended by Masimo (Masimo Corporation CA, USA).

Authors' contributions. CF, TC, BL, JCMR designed the study protocol, analysed the data, and drafted the manuscript. CF was the major contributor to the experiments and in writing the manuscript. DJ, YL, JCMR, BB, MR participated in a part of the experiments. CF, BBconducted the data extraction and data management. FER, CF generated the statistics. EL did all the histology analysis. All the authors read and approved the final manuscript.

Acknowledgements. We are thankful to the technicians of the Surgery School at Lorraine University, namely Aude Falanga,Vanessa Marie, Fréderique Groubatch, Brice Mourer for their professionalism and constant attention. We are thankful to Malha Sadounefor biology support.

\section{References}

1. Cordioli RL, Lyazidi A, Rey N, Granier J-M, Savary D, Brochard L, et al. Impact of ventilation strategies during chest compression. An experimental study with clinical observations. J Appl Physiol. 2016 Jan 15;120(2):196-203.

2. Cordioli RL, Brochard L, Suppan L, Lyazidi A, Templier F, Khoury A, et al. How Ventilation Is Delivered During Cardiopulmonary Resuscitation: An International Survey. Respir Care. 2018 May 8.

3. Cordioli RL, Grieco DL, Charbonney E, Richard J-C, Savary D. New physiological insights in ventilation during cardiopulmonary resuscitation. Curr Opin Crit Care. 2019 Feb;25(1):37-44.

4. Monsieurs KG, Nolan JP, Bossaert LL, Greif R, Maconochie IK, Nikolaou NI, et al. European Resuscitation Council Guidelines for Resuscitation 2015: Sect. 1. Executive summary. Resuscitation. 2015 Oct;95:1-80.

5. Link MS, Berkow LC, Kudenchuk PJ, Halperin HR, Hess EP, Moitra VK, et al. Part 7: Adult Advanced Cardiovascular Life Support: 2015 American Heart Association Guidelines Update for Cardiopulmonary Resuscitation and Emergency Cardiovascular Care. Circulation. 2015 Nov 3;132(18 Suppl 2):S444464.

6. Idris AH. Reassessing the need for ventilation during CPR. Ann Emerg Med. 1996 May;27(5):569-75.

7. Bernhard M, Hossfeld B, Kumle B, Becker TK, Böttiger B, Birkholz T. Don't forget to ventilate during cardiopulmonary resuscitation with mechanical chest compression devices. Eur J Anaesthesiol. 2016;33(8):553-6.

8. Grieco DL, Brochard J, Drouet L, Telias A, Delisle I, Bronchti S. G, et al. Intrathoracic Airway Closure Impacts CO2 Signal and Delivered Ventilation during Cardiopulmonary Resuscitation. Am J Respir Crit Care Med. 2019;15(6):728-37. 199(.

9. Branditz FK, Kern KB, Campbell SC. Continuous transtracheal oxygen delivery during cardiopulmonary resuscitation. An alternative method of ventilation in a canine model. Chest. 1989 Feb;95(2):441-8.

10. Saïssy JM, Boussignac G, Cheptel E, Rouvin B, Fontaine D, Bargues L, et al. Efficacy of continuous insufflation of oxygen combined with active cardiac compression-decompression during out-of-hospital cardiorespiratory arrest. Anesthesiology. 2000 Jun;92(6):1523-30.

11. Bertrand C, Hemery F, Carli P, Goldstein P, Espesson C, Rüttimann M, et al. Constant flow insufflation of oxygen as the sole mode of ventilation during outof-hospital cardiac arrest. Intensive Care Med. 2006 Jun;32(6):843-51.

12. Georges Boussignac F, Galia H, Roy M, Blanche J, Oudet J-C, Deslandes, et al. Comparative testing of a No-No Flow new ventilation device during experimental cardio pul- monary resuscitation on a lung-simulation system. Resuscitation. 2015;96:49.

13. Vygon. Vygon launches b-card, an innovative device that combines continuous chest compressions and dynamic oxygenation for CPR. Vygon; 2016 https://www.vygon.com/wp-content/uploads/2016/09/160808-vygon-b- card-en-final.pdf.

14. Moore JC, Lamhaut L, Hutin A, Dodd KW, Robinson AE, Lick MC, et al. Evaluation of the Boussignac Cardiac arrest device (B-card) during cardiopulmonary resuscitation in an animal model. Resuscitation. 2017;119:81-8.

15. Fritz C, Kimmoun A, Vanhuyse F, Trifan BF, Orlowski S, Falanga A, et al. High Versus Low Blood-Pressure Target in Experimental Ischemic Prolonged Cardiac Arrest Treated with Extra Corporeal Life Support. Shock. 2017 Jun;47(6):759-64.

16. West JB. Respiratory physiology: The Essentials 2012, 9 ed., Lippincott Williams \& Wilkins, 24 - 5. In.

17. Wang $\mathrm{C}-\mathrm{H}$, Chang W-T, Huang C-H, Tsai M-S, Yu P-H, Wang A-Y, et al. The effect of hyperoxia on survival following adult cardiac arrest: a systematic review and meta-analysis of observational studies. Resuscitation. 2014 Sep;85(9):1142-8.

18. Douzinas EE, Patsouris E, Kypriades EM, Makris DJ, Andrianakis I, Korkolopoulou P, et al. Hypoxaemic reperfusion ameliorates the histopathological changes in the pig brain after a severe global cerebral ischaemic insult. Intensive Care Med. 2001 May;27(5):905-10.

19. Roberts BW, Kilgannon JH, Hunter BR, Puskarich MA, Pierce L, Donnino M, et al. Association Between Early Hyperoxia Exposure After Resuscitation From Cardiac Arrest and Neurological Disability: Prospective Multicenter Protocol-Directed Cohort Study. Circulation. 2018;15(20):2114-24. 137(.

20. Skrifvars MB. Towards interventional trials on the use of oxygen during and after cardiac arrest. Resuscitation. 2016 Apr;101:A3-4.

21. Humaloja J, Litonius E, Efendijev I, Folger D, Raj R, Pekkarinen PT, et al. Early hyperoxemia is not associated with cardiac arrest outcome. Resuscitation. 2019 Jul;140:185-93. 
22. Schneider AG, Eastwood GM, Bellomo R, Bailey M, Lipcsey M, Pilcher D, et al. Arterial carbon dioxide tension and outcome in patients admitted to the intensive care unit after cardiac arrest. Resuscitation. 2013 Jul;84(7):927-34.

23. Eastwood GM, Schneider AG, Suzuki S, Peck L, Young H, Tanaka A, et al. Targeted therapeutic mild hypercapnia after cardiac arrest: A phase II multicentre randomised controlled trial (the CCC trial). Resuscitation. 2016;104:83-90.

24. Dyson A, Stidwill R, Taylor V, Singer M. The impact of inspired oxygen concentration on tissue oxygenation during progressive haemorrhage. Intensive Care Med. 2009 Oct;35(10):1783-91.

25. van den Brule JMD, van der Hoeven JG, Hoedemaekers CWE. Cerebral Perfusion and Cerebral Autoregulation after Cardiac Arrest. Biomed Res Int. 2018;2018:4143636.

26. Schnaubelt S, Sulzgruber P, Menger J, Skhirtladze-Dworschak K, Sterz F, Dworschak M. Regional cerebral oxygen saturation during cardiopulmonary resuscitation as a predictor of return of spontaneous circulation and favourable neurological outcome - A review of the current literature. Resuscitation. 2018 Apr;125:39-47.

27. Hodgkin BC, Lambrew CT, Lawrence FH, Angelakos ET. Effects of PEEP and of increased frequency of ventilation during CPR. Crit Care Med. 1980 Mar;8(3):123-6.

28. Voelckel WG, Lurie KG, Zielinski T, McKnite S, Plaisance P, Wenzel V, et al. The effects of positive end-expiratory pressure during active compression decompression cardiopulmonary resuscitation with the inspiratory threshold valve. Anesth Analg. 2001 Apr;92(4):967-74.

29. McCaul C, Kornecki A, Engelberts D, McNamara P, Kavanagh BP. Positive end-expiratory pressure improves survival in a rodent model of cardiopulmonary resuscitation using high-dose epinephrine. Anesth Analg. 2009 Oct;109(4):1202-8.

30. Charbonney E, Delisle S, Savary D, Bronchti G, Rigollot M, Drouet A, et al. A new physiological model for studying the effect of chest compression and ventilation during cardiopulmonary resuscitation: The Thiel cadaver. Resuscitation. 2018;125:135-42.

\section{Figures}

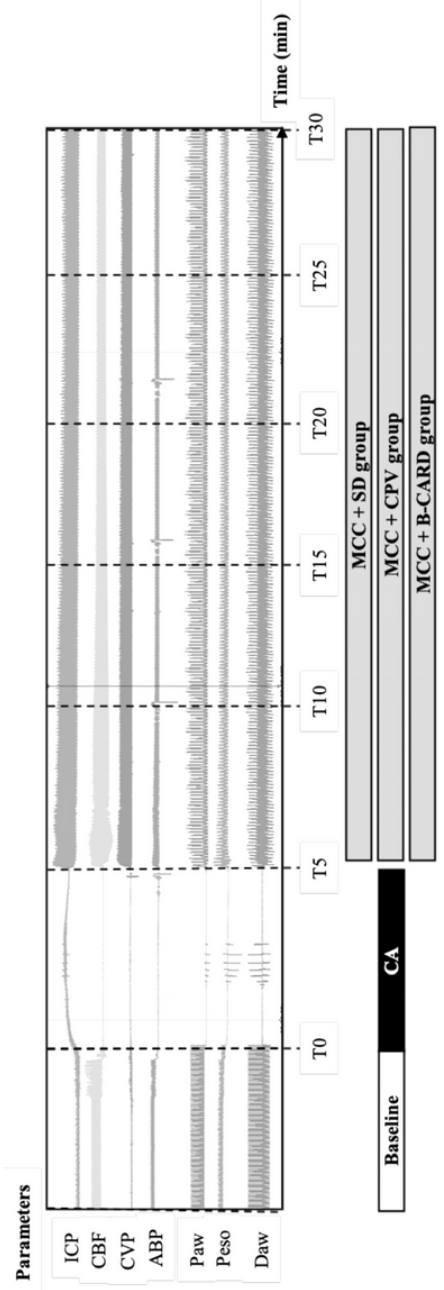

Figure 1

Overall protocol time table and illustration of the cure obtained. CA: cardiac arrest; CVP: central venous pressure; Paw: Airway Pressure; Peso: esophageal Pressure; Daw: Airway Flow; CPR: Cardio-Pulmonary Resuscitation. SD: Standard ventilation Group; CPV: Cardio-Pulmonary Ventilation Group; BC: B-CARD 
Group; MAP: Mean Arterial Pressure.
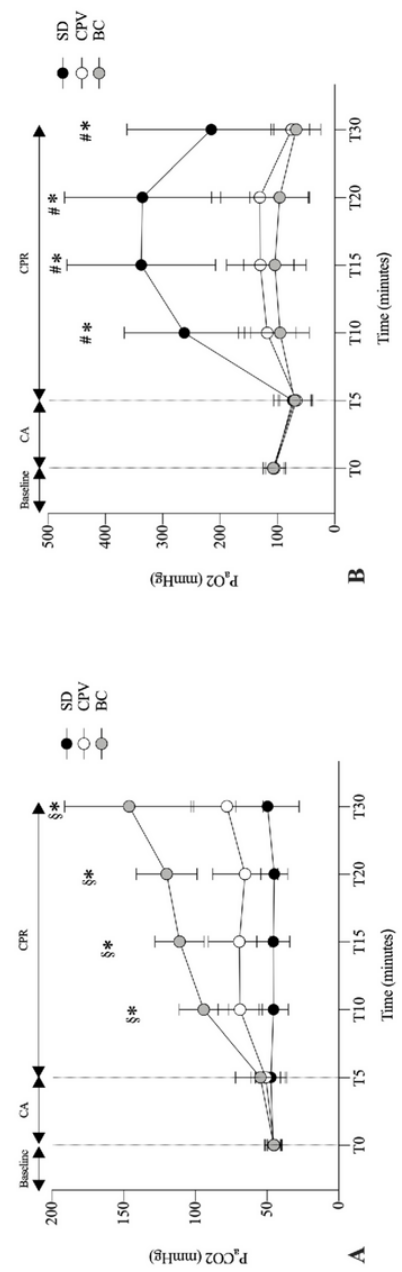

\section{Figure 2}

Blood gas evolution according to the ventilatory mode randomization. A: PaCO2 arterial serum level of $\mathrm{CO} 2$ (carbon dioxide partial pressure) evolution; $\mathrm{B}$ : $\mathrm{PaO} 2$ arterial serum level of $\mathrm{O} 2$ (oxygen partial pressure) evolution; SD: Standard ventilation Group; CPV: Cardio-Pulmonary Ventilation Group; BC: B-CARD Group. Values are given as mean \pm SD. The sign displays the bonferroni corrected p-values from pairwise comparisons between groups performed by the Dunn's post hoc test: \# SD vs. CPV, * SD vs. BC, § CPV vs. BC 

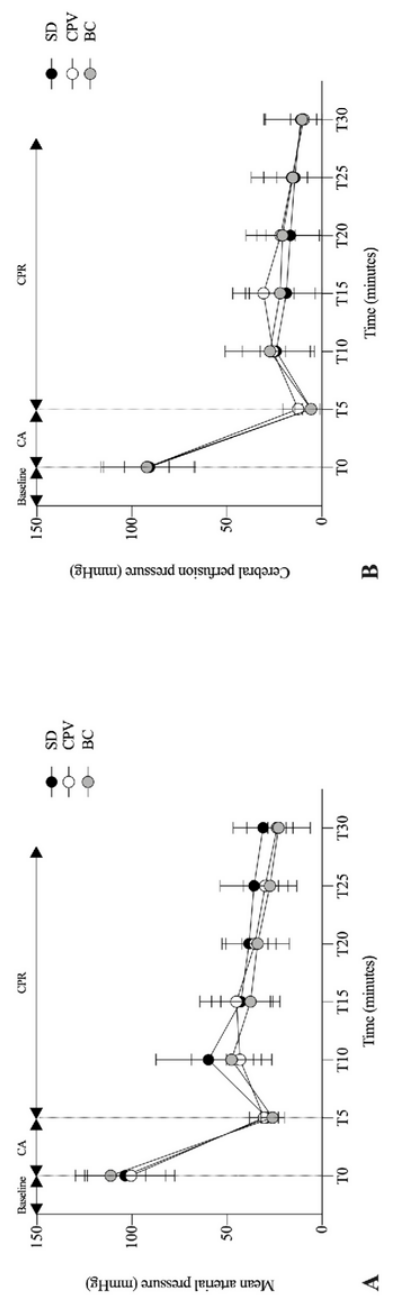

\section{Figure 3}

Macro-haemodynamic parameters evolution during the protocol evolution. A: mean arterial pressure evolution according to the tree groups; B: cerebral perfusion pressure (CePP) evolution. CePP is calculate as mean arterial pressure minus intracranial pressure. SD: Standard ventilation Group; CPV: CardioPulmonary Ventilation Group; BC: B-CARD Group. Values are given as mean \pm SD. Kruskal-Wallis one-way analysis of variance was used to compare continuous variables, p-values from pairwise comparisons between groups performed by the Dunn's post hoc test: \# SD vs. CPV, * SD vs. BC, § CPV vs. BC. 

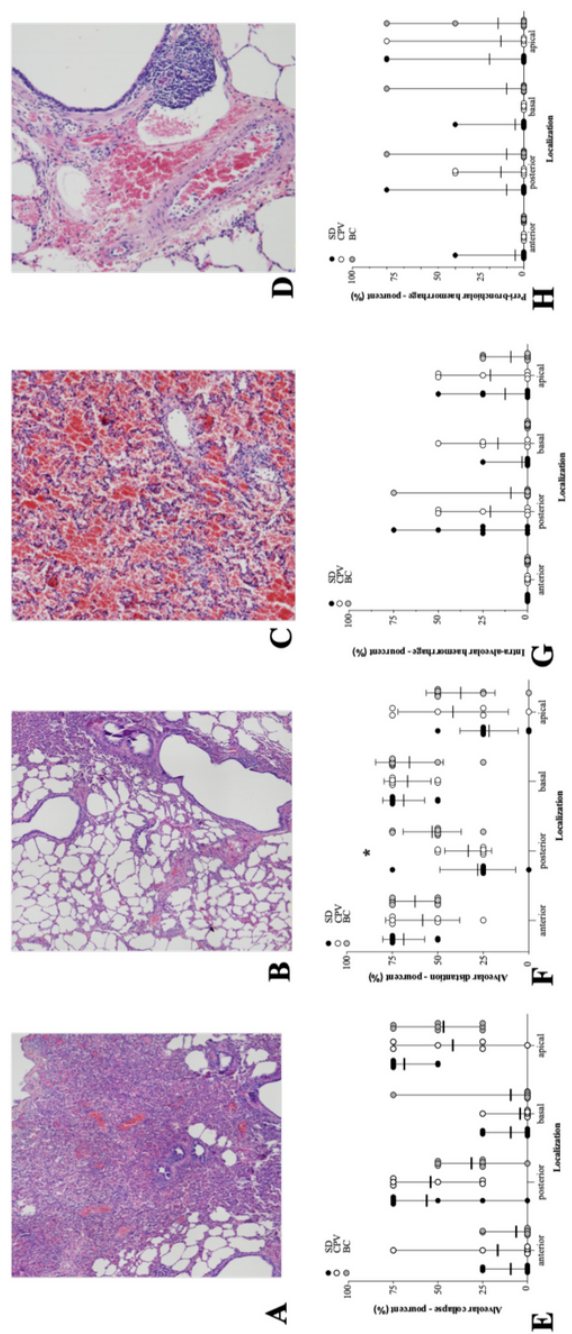

\section{Figure 4}

Post mortem histology analysis of lung injury. A: histology of collapse; B: histology of distension; C: histology of intra-alveolar haemorrhage; D: histology of peri-bronchiolar haemorrhage; E: pencentage of alveolar collapse regarding localization; F: pencentage of alveolar distention regarding localization; G: pencentage of intra-alveolar haemorrhage regarding localization; $\mathrm{H}$ : pencentage of peri-bronchiolar haemorrhage regarding localization. SD: Standard ventilation Group; CPV: Cardio-Pulmonary Ventilation Group; BC: B-CARD Group

\section{Supplementary Files}

This is a list of supplementary files associated with this preprint. Click to download.

- ESMTable1.1.docx

- ESMTable1.2.docx 